\title{
Diagnostic Imaging and Different Modalities of Treatment of Temporomandibular Joint Dysfunction
}

\author{
Waleed saber*, Msc, Mohamed Shoeib*, MD, Sherif Bakri*, Ahmed Gaber* \\ ,MD, Mohamed Abdelaal*, MD , Alaa A. Redwan**, MD \\ *Plastic Surgery Department, Sohag University Hospital, Faculty of Medicine, Sohag \\ University, Egypt \\ ** General Surgery Department, Sohag University Hospital, Faculty of Medicine, \\ Sohag University, Sohag, Egypt \\ Correspondent author: \\ Sherif Bakri: Lecturer of Plastic Surgery, Faculty of Medicine, Sohag University \\ Sohag, Egypt tel: +201003332114 Email: bakrisherif@gmail.com
}

\section{Abstract \\ Introduction:}

Temporomandibular joint dysfunction (TMD) is a common condition that affects up to $39 \%$ of the population and associated with a wide range of clinical signs and symptoms such as, pain, clicking, crepitation, restriction of movement, deviation of the jaw, headache, vertigo, and tinnitus. The etiology of TMD is of multifactorial origin like trauma, parafunction, malocclusion and degenerative joint diseases.

\section{Materials and Methods}

This study included 18 patients who had symptoms of temporomandibular joint dysfunction. The patients were evaluated in the plastic surgery department at Sohag University Hospital.

\section{Results}

The study conducted on 18 patients; 8 male and 10 female, the mean age is 26 years ranging from 17 to 40 years.

From the 18 patients; 2 cases received no treatment and 16 patients ( 7 males and 9 females) had received several modalities of treatment.

In this study we used a variety of modalities of treatment such as, medical treatment to 8 cases, relaxation to 1 case, splint to 2 cases, arthrocentesis to 1 case, arthroscopy to 2 cases, discectomy for 1 case and arthroplasty for 1 case.

\section{Introduction}

Temporomandibular joint dysfunction (TMD) is a common condition that affects up to $39 \%$ of the population and associated with a wide range of clinical signs and symptoms such as, pain, clicking, crepitation, restriction of movement, deviation of the jaw, headache, vertigo, and tennitus (1).

The etiology of TMD is of multifactorial origin like trauma, parafunction, malocclusion and degenerative joint diseases (2). Temporomandibular joint dysfunction expressed pathologically such as, internal derangement, osteoarthritis, and joint effusion (3). Magnetic resonance imaging is the primary imaging technique in the diagnosis of dysfunction, because it provides superior information of all joint structures in non-invasive way (4).

Anterior disc displacement is one of the common radiological findings of TMDs and the most common cause of joint sounds (5). It is in symptomatic individuals is much higher than in the normal population, disc displacement has been found in $77 \%$ - 94\% of patients with TMD symptoms referred for MRI and $20 \%-34 \%$ of the asymptomatic population (6). 
Our goal of treatment is to improve symptoms such as, pain and alteration of function as mastication and speech which can greatly affect mouth health care and quality of life (7).

Management of TMDs can be divided to non-invasive, minimally invasive, and invasive modalities, finally, in end stage disease; salvage modalities as arthroplasty and joint replacement are considered (8).

We tried to make a good diagnosis by the proper imaging and selection of the proper line of treatment for joint protection, improvement of symptoms and preservation of joint function. In this article we have described our work of one year on TMD patients and we have reported the importance of proper imaging and treatment of temporomandibular joint dysfunction.

\section{Results}

The study conducted on 18 patients; 8male and 10 female, the mean age is 26 years ranging from 17 to 40 years (table 1 ).

CT and MRI were done to the cases; CT was done for 2 cases with history of conylar fractures showing: the first case with bilateral bony ankylosis, the second case was fibrous ankylosis.

MRI is useful for demonstration old disc position and morphology, osteoarthritis, joint effusion and condylar translation, it was done to 10 patients with variety of findings, as regard disc position; 2 cases with normal disc position, 6 cases with anterior disc displacement with reduction and 2 cases with anterior disc displacement without reduction.

As regard disc shape; 3 cases with normal disc morphology and 7 cases with changes in disc morphology and diffuse thickening.

As regard disc mobility; 6 cases with normal disc mobility, 3 cases with limited disc movement, and a case with stuck disc.

Osteoarthritis in 4 cases and 6 patients with joint effusion and bone marrow edema (table 2).

\section{Treatment modalities}

From the 18 patients; 2 cases received no treatment and 16 patients ( 7 males and 9 females) had received several modalities of treatment.

In this study we used a variety of modalities of treatment such as, medical treatment to 8 cases, relaxation to 1 case, splint to 2 cases, arthrocentesis to 1 case, arthroscopy to 2 cases, discectomy for 1 case and arthroplasty for 1 case (table 3 ).

Medical treatment was given to 8 patients, 4 males and 4 females. Analgesics, muscle relaxant and steroids for 2 weeks.

Masseteric hypertrophy was reported in 1 case underwent relaxation and passive movement of the joint

Splint therapy was done to 2 cases with medical treatment. 
Discectomy was done to 1 case with post operative pain improvement.

Arthroplasty with interposition temporalis fascia was done to 1 case.

Arthrocentesis was done to a female patient with marked limitation of jaw movement's pain, resulted in marked improvement in movement and pain relief. Arthroscopy was done to 2 cases with lysis and lavage of the joint (figure 1).

\section{Representative cases}

\section{Case No.1:}

Female patient, 17 years old presented with pain and click on the Lt TMJ of 3months. MRI was done to the patient showing osteoarthritic Lt TMJ with anterior disc displacement with reduction. Arthroscopic lysis and lavage and injection of muscle relaxant on the lateral pterygoid muscle were done to the patient (figure 2).

\section{Case No.2:}

Male patient, 35 years with over jet and CT face was done showing bilateral bony ankylosis. Arthroplasty was done with temporalis fascia flap to fill the dead space (figure 3).

\section{Discussion}

Temporomandibular joint dysfunction is a common condition affects up to $39 \%$ of population associated with a wide range of clinical symptoms and signs such as, pain, clicking, restriction of mouth opening, jaw deviation, tinnitus, headache, vertigo (1).

A wide spectrum of
temporomandibular joint dysfunction types and causes according to RDC/TMD research diagnostic criteria, a dual axis system. We can differentiate the patients with axis 1 is a clinical diagnostic system to 3 groups, muscle group, derangement group, and degenerative group. Axis 2 is a psychosocial evaluation that assess pain scale, pain related disability, depression, and non specific physical symptoms (9).

In our study we choose the imaging procedure according the pathology, we did CT scan in two cases of ankylosis. CT is mainly used for bony lesions, ankylosis, and neoplasia.

MRI is the primary imaging technique in diagnosis of TMJ abnormalities; it gives superior information for all joint structures in a non invasive way (10). MRI was done for 10 cases in our study many findings were detected. MRI confirmed disc displacement and internal derangement in 8 of the 10 patients $(80 \%)$. This observation compares that with other studies that revealed the percentage of disc displacement and internal derangement among symptomatic patients ranging from $64.4 \%$ to $89 \%$. It had been proven that MRI is the golden standard technique in diagnosis of several TMJ pathologies. Many studies had confirmed that issue (11).

Osteoarthritis was found in $40 \%$ of the cases that match the literature with a percentage of $11 \%$ to $59 \%$ of symptomatic patients (12). Effusion is a finding that easily can be detected by MRI (13).

A drawback was that studies had been done on MRI in diagnosing joint pathologies only concentrated on disc displacement and morphology and with no attention about bone marrow, temporal segment of the joint and joint effusion (14).

Different modalities of treatment were used in our study in order to find a suitable and reliable line of management. It was attributed to the type and degree of the dysfunction. Usually the treatment begins with medical treatment, non surgical options and the surgery is the last option. The majority of TMD patients can be successfully treated by non-surgical 
therapies and surgical interventions may be required for only a few number of TMD population. All non-surgical treatment options must be exhausted before undertaking the invasive methods for the management of TMD (15).

In 8 patients of the study medical treatment was used $44.5 \%$ of the cases. Medical treatment should be used according to a schedule rather than needed for pain relief to obtain good result. We used it for 2 weeks. NSAIDs were mainly for pain control and can be used as initial line of treatment or in combination with other lines. Muscle relaxants are very important line to rest the muscles and relieve muscle spasm. Relaxation regimens in chronic pain are very effective for the patient and, with self care and awareness of the conditions that affect the joints, can change the behavior of the patient and decrease the pain. In our study a case with bilateral massetteric hypertrophy, self care and relaxation advised.

Splints are plastic mouth pieces that are fitted in the upper and lower jaw for bite blockade minimize teeth attrition, used in control of bruxism to prevent muscle strain, fatigue, fibrosis, capsulitis, and adhesions (16). It was very effective in reducing pain and decrease muscle spasm through bite correction. Many study had a debate on the long term use of the splints (17). In our study 2 cases with internal derangement splints and medical treatment were given, and the role of splint was proved.

Arthrocentesis with joint lavage considered the simplest surgical intervention that is very effective in reducing pain, joint sound, and improves mouth opening (18). The improvement in jaw function explained by that arthrocentesis loosen the adherent disc, removing inflammatory mediators, so nutrient perfusion will be corrected resulting in free disc mobility and so improved mouth opening (18). In our study there is a case of internal derangement and severe limitation of mouth opening. After arthrocentesis and lavage of the joint marked improvement of mouth opening and pain relief occurred.

Discectomy used for removal of the damaged painful and displaced articular disc. It is used as a minor procedure instead of joint replacement. One drawback is after removal of the disc the joint loading increase during jaw movement (19). In our study, discectomy was done to a female patient with pain not responding to medical treatment. Firstly pain disappear completely postoperatvely unfortunately, the patient experience recurrence of pain after 10 days postoperative with jaw movement due to increase joint loading.

Arthroscopy was used to remove inflamed tissues, lavage and lysis, realignment of the disc and condyle (20). Arthroscopy is considered a surgery to come back the displaced disc in its anatomical position. This procedure is done through a small incision which is made in front of the patient's ear with small, thin instrument that contain a lens and light source (15).

Arthroscopy has a beneficial role in detecting the osteoarthritic changes in the joint (21). Arthroscopy considered a safe, minimal invasive surgical procedure, authors reported some complications such as, infection, ear complications, neurological, or vascular injuries and perforation of middle cranial fosa (22).

In our study arthroscopic lysis and lavage of the joint done to 2 cases with detection and confirmation of the presence of osteoarthritis and synovitis. There were no complications reported in our cases and improvement of pain and mouth opening regardless 
numbness at the site of puncture in one case and resolve completely after 3 weeks. With injection of hyaluronic acid in the joint.

Ankylosis of the temporomandibular joint may be fibrous or bony. That cause jaw movement restriction and may end with no movement at all if neglected which is the sequel of neglected trauma, surgery, infection. Temporomandibular disc damage, prolonged immobilization of the mandible after inracapsular trauma. Other causes myositis ossificans, osteochondroma, rheumatoid arthritis and ankylosing spondylitis (22).

MR arthrography useful for fibrous adhesion evaluation. CT scan is necessary when bony ankylosis is suspected (23). In our study, male patient 35 years old with bilateral bony ankylosis, condylectomy was done and resection of the ankylotic mass was done. Mouth opening markedly improved. The incisal opening preoperatively was over jet and postoperatively admitted 2 fingers. Arthroplsty with resection of the ankylotic mass and recontouring of the dead space with temporalis muscle fascia. Total joint replacement has the advantage of immediate function, facial asymmetry and bone defect correction and lack of donor site morbidity. It reported that it reduce incidence of reankylosis (24). Long term studies of total joint implants have been reported to ensure significant increase in life score and durability without the presence of foreign body reaction (25). In our study there were no cases because joint

\section{References}

1. Aiken A, Bouloux G, Hudgins P. MR imaging of the temporomandibular joint. Magn Reson Imaging Clin N Am 2012; 20: 397-412.

2. Greenberg MS, Glick M, Slip J. Burket's oral medicine, diagnosis and replacement needs a high cost and facilities.

MRI and CT greatly helping us in diagnosis of TMJ dysfunction. It is confirmed that MRI is the gold standard method for diagnosis in a non invasive way, it give information about disc position, disc morphology, osteoarthritis, effusion and bone marrow edema. CT mainly used in case of ankylosis, bony lesions, and neoplasm. Our study greatly matches the world statistics that the main line of treatment is the medical treatment followed by the least invasive method and finally the invasive method (15). But we need further studies and large samples of patients for more explanation and clarification of treatment modalities.

\section{Conclusion}

Imaging of the TMJ pathology guided us for proper and correct management. There are a wide variety of imaging modalities for the temporomandibular joint dysfunctions. MRI is considered the gold standard imaging tool for diagnosis of the dysfunction in a simple and perfect way without intervention. It gives superior information about joint structures, including disc morphology and position. Effusion, bone marrow edema, and osteoarthritis also detected. CT imaging is most helpful in diagnosis of bony lesions, ankylosis, and neoplasm.

Treatment of temporomandibular joint expresses a very wide variety of modalities, ranging from the self care and rest up to the complicated surgical procedures as joint replacement

treatment. Hamilton: Decker; 2008. p. 223-57.

3. Maizlin ZV, Nutiu N, Dent PB, et al. Displacement of the temporomandibular joint disk: correlation between clinical findings 
and MRI characteristics. J Can Dent Assoc. 2010; 76:1-5.

4. Orlando B, Chiappe G, Landi N, et al. Risk of temporomandibularjoint effusion related to magnetic resonance imaging signs of disc displacement. Med Oral Patol Oral Cir Bucal 2009; 14:188-19.

5. Bakke M, Møller E, Werdelin L, Dalager T,Kitai N, Kreiborg S.Treatment of severe temporomandibular joint clicking with botulinum toxin lateral pterygoid muscle in two cases of anterior disc displacement. Oral Surg Oral Med Oral Pathol Oral RadiolEndod. 2005; 100: 693-700.

6. Tomas X, Pomes J, Berenguer J, et al. MR imaging of temporomandibular joint dysfunction: a pictorial review. Radiographics2006; 26:765-81.

7. Vasilki Karlis, Robert Glickman. Non surgical management of Temporomandibular Disorders. In, Michael Miloro(Ed). Peterson $\mathrm{s}$ principles of maxillofacial surgery, second edition. London, BC Decker Inc, 2004; 950.

8. Mercuri LG (2006). Surgical management of TMJ arthritis. In: TMDs, an evidence-based approach to diagnosis and treatment. Laskin DM, Greene CS, Hylander WL, editors. Chicago: Quintessence, pp. 455-468.

9. Barclay P, Hollender LG, Maravilla $\mathrm{KR}$, et al. Comparison of clinical and magnetic resonance imaging diagnosis in patients with disk displacement in the temporomandibular joint. Oral Surg Oral Med Oral Pathol Oral Radiol Endod 1999; 88:37.

10. Westesson PL O-YM, Sano T, Okano T. Anatomy, Pathology, and Im aging of the Temporomandibular Joint. In: Som PM, Curtin HD, ed. Head and Neck Imaging. St. Louis: Mosby, 2011: 1547-613.
11. Westesson PL, Katzberg RW, Tallents RH et al.: Temporomandibular joint: comparison of MR images with cryosectional anatomy. Radiology 1987; 164:59.

12. Emshoff R, Brandlmaier I, Gerhard $S$, et al. Magnetic resonance imaging predictors of temporomandibular joint pain. J Am Dent Assoc. 2003; 134:705-714.

13. Zhang S, Yang C, Chen $M$ et al.: Magnetic resonance imaging in the diagnosis of intra-articular adhesions of the temporomandibular joint. Br J Oral Maxillofac Surg 2009; 47:389.

14. Choi YS, Asaumi J, Hisatomi M et al.: Analysis of magnetic resonance images of disk positions and deformities in 1,265patients with temporomandibuar disorder.open dent j 2009; 3:1.

15. Ingawale Shirish and Tarun Goswami. Temporomandibular Joint: Disorders, Treatments, and Biomechanics. Annals of Biomedical Engineering 2009; 37: 976-996.

16. Chung, S. C., Y. K. Kim, and H. S. Kim. Prevalence and patterns of nocturnal bruxofacets on stabilization splints in temporomandibular disorder patients. Cranio.2000; 18:92.

17. Raphael, K. G., J. J. Marbach, J. J. Klausner, et al. Is bruxism severity a predictor of oral splint efficacy in patients with myofascial face pain? J. Oral Rehabil.2003; 30:17-29.

18. Kuruvilla V. E and K. Prasad. Arthrocentesis in TMJ Internal Derangement: A Prospective StudyJ. Maxillofac. Oral Surg. 2012; 11:5356.

19. Tanaka E, Detamore MS, Mercuri LG. Degenerative disorders of the temporomandibular joint: etiology, diagnosis, and treatment. J Dent Res 2008; 87: 296-307.

20. Cleveland Clinic. Health information. Retrieved on 09/21/ 2007, from http://www.clevelandclinic.org/health/. 
patients with temporomandibular disorder. Open Dent J 2009; 3:1

21. White RD: Arthroscopy of the temporomandibular joint: technique and operative images. Atlas Oral Maxillofac Surg Clin North Am 2003; 11:129.

22. Gundlach KK: Ankylosis of the Temporomandibular joint. J CranioMaxillo-Fac Surg, 2010; 38: 122-30.

23. Cannizzaro E, Schroeder S, Müller LM, et al. Temporomandibular joint involvement in children with juvenile

\section{Legends}

Figure 1

Modalities of treatment chart

Figure 2

Sixteen years old female patient with pain and click on the Lt TMJ, arthroscopy was done.

A, the patient preoperative, $\mathrm{B}$; the patient is postoperative,

Figure 3

Thirty five years old male patient with locking and failure of mouth opening, CT showing bilateral bony ankylosis of TMJs

A, the patient preoperative, B; the patient is postoperative,

\section{Table 1}

Demoghraphic data of the patients

Table 2

MRI findings of the patients

Table 3

Treatments modalities

Figure 1 
SOHAG MEDICAL JOURNAL

Vol. 21 No.3 october 2017

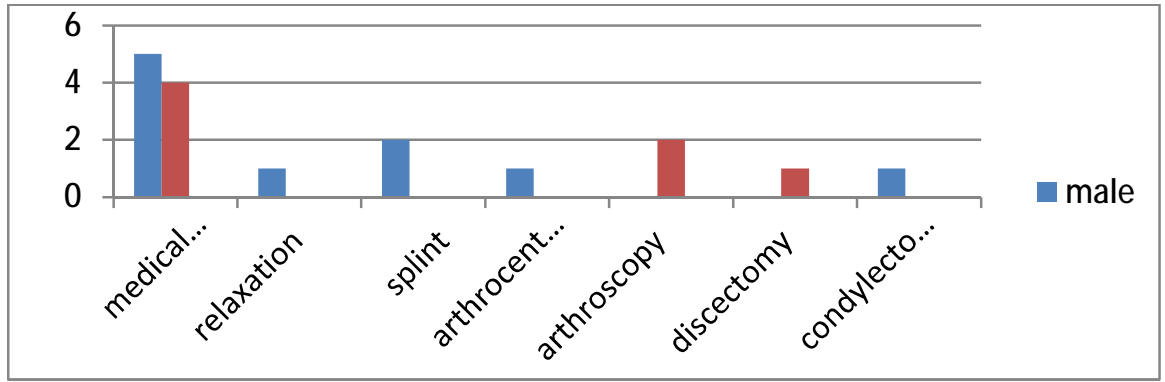

Figure 2

A

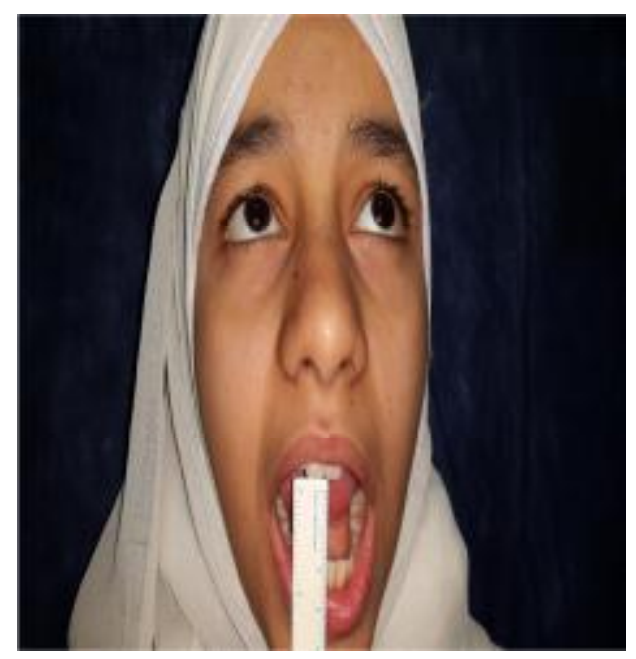

Figure 3

A

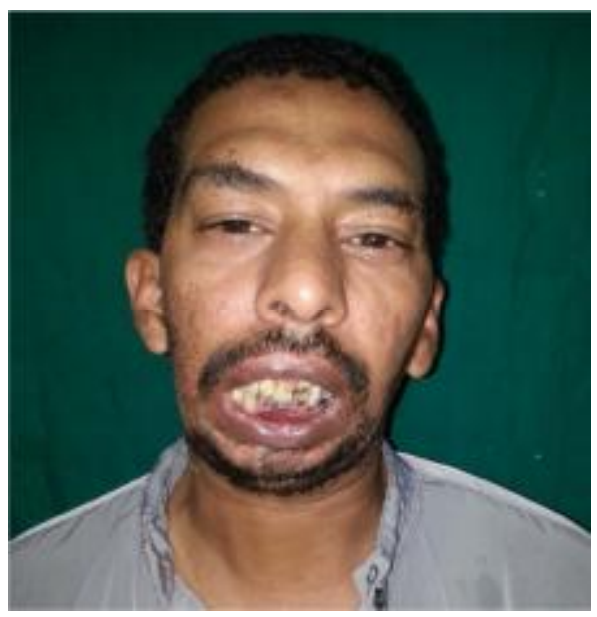

B

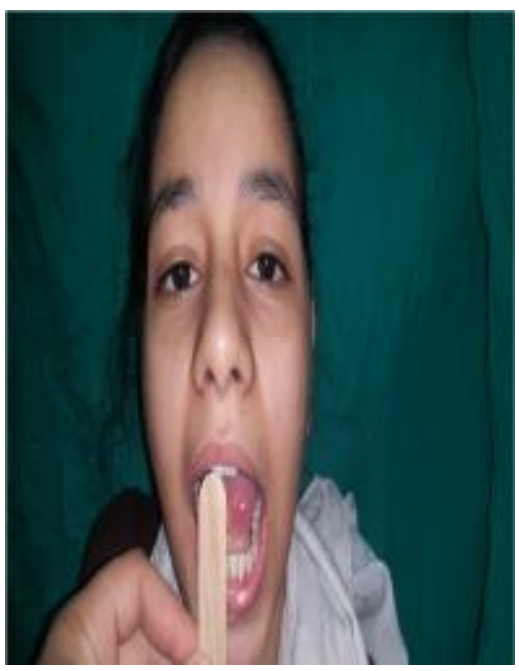

B

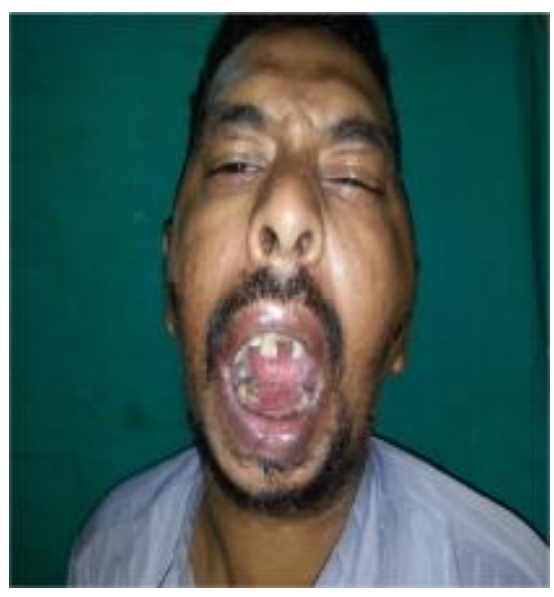


SOHAG MEDICAL JOURNAL Diagnostic Imaging and Different Modalities of Treatment

Table 1

\begin{tabular}{||r|r|}
\hline Patient group & Demographic data \\
No. 18 & Age in years \\
$(17-40)$ & Range (Min-Max): \\
26 & Mean: \\
\hline \hline & Sex \\
$8(44.5 \%)$ & Male: No. $(\%)$ \\
$10(55.5 \%)$ & Female: No. $(\%)$ \\
\hline \hline $2(11.1 \%)$ & Side \\
$6(33.4 \%)$ & Right : No (\%) \\
$10(55.5)$ & Left : No (\%) \\
\hline \hline
\end{tabular}

Table 2

\begin{tabular}{|c|c|}
\hline $\begin{array}{r}\text { Patients group } \\
10 \text { of } 18 \\
\end{array}$ & MRI finding \\
\hline $\begin{array}{ll}2 & (20 \%) \\
6 & (60 \%) \\
2 & (20 \%)\end{array}$ & $\begin{array}{r}\text { Disc postion } \\
\text { Normal no \% } \\
\text { ADDWR no \% } \\
\text { ADDWOR no \% }\end{array}$ \\
\hline $\begin{array}{ll}3 & (30 \%) \\
7 & (\mathbf{7 0 \%}) \\
\end{array}$ & $\begin{array}{r}\text { Disc morphology } \\
\text { Normal } \\
\text { Abnormal }\end{array}$ \\
\hline $\begin{array}{ll}6 & (60 \%) \\
3 & (30 \%) \\
1 & (10 \%)\end{array}$ & $\begin{array}{r}\text { Disc mobility } \\
\text { Normal } \\
\text { Limited } \\
\text { Stuck }\end{array}$ \\
\hline $\begin{array}{ll}4 & (40 \%) \\
6 & (60 \%)\end{array}$ & $\begin{array}{r}\text { OA } \\
\text { Present no \% } \\
\text { Absent no \% }\end{array}$ \\
\hline $\begin{array}{ll}1 & (10 \%) \\
9 & (90 \%)\end{array}$ & $\begin{array}{r}\text { Bone marrow edema } \\
\text { Present no \% } \\
\text { Absent no \% }\end{array}$ \\
\hline $\begin{array}{ll}6 & (60 \%) \\
4 & (40 \%)\end{array}$ & $\begin{array}{r}\text { Effusion } \\
\text { Present no \% } \\
\text { Absent No \% }\end{array}$ \\
\hline
\end{tabular}


SOHAG MEDICAL JOURNAL

Vol. 21 No.3 october 2017

Table 3

\begin{tabular}{|c|c|}
\hline $\begin{array}{r}\text { Patient group } \\
\text { No. }(\%)\end{array}$ & Treatment modalities \\
\hline Male 4(25\%) & \multirow[t]{2}{*}{ Medical treatment } \\
\hline Female $4(25 \%)$ & \\
\hline Male $1(6.25 \%)$ & \multirow[t]{2}{*}{ Relaxation } \\
\hline Female 0 & \\
\hline Male 0 & Splint \\
\hline \multicolumn{2}{|l|}{ Female $2(12.5 \%)$} \\
\hline Male 0 & \multirow[t]{2}{*}{ Arthrocentesis } \\
\hline Female $1(6.25 \%)$ & \\
\hline Male $\mathbf{0}$ & \multirow[t]{2}{*}{ Arthroscopy (diagnostic and therapeutic) } \\
\hline Female $2(12.5 \%)$ & \\
\hline Male 0 & \multirow[t]{2}{*}{ Discectomy } \\
\hline Female $1(6.25 \%)$ & \\
\hline $\begin{array}{ll}\text { Male } & 1(6.25 \%) \\
& \text { Female } 0\end{array}$ & \multirow{2}{*}{$\begin{array}{l}\text { Condylectomy with arthroplasty with } \\
\text { interpostioning temporalis fascia flap }\end{array}$} \\
\hline Female 0 & \\
\hline
\end{tabular}

